\title{
From Fossils to Function: Integrative and Taxonomically Inclusive Approaches to Vertebrate Evolutionary Neuroscience
}

\author{
Ashley C. Morhardt \\ Department of Neuroscience, Washington University School of Medicine, St. Louis, MO, USA
}

The 29th annual Karger Workshop, entitled "From Fossils to Function: Integrative and Taxonomically Inclusive Approaches to Vertebrate Evolutionary Neuroscience," held in Hyattsville, MD, USA, in 2017, brought together international experts to provide an overview of the practices, pitfalls, and future aims of paleoneurology within the broader context of evolutionary neuroscience. Workshop talks, group discussions, and related papers in this special issue of Brain, Behavior, and Evolution (BBE) spanned three major topics: (1) best practices for the inference, reconstruction, and comparative investigation of endocranial soft-tissue structures in extinct vertebrate taxa, (2) comparative studies of brains in a wide array of extant taxa, with specific focus on groundbreaking structural and functional neuroimaging, and (3) moving towards research that embraces an integrative approach (i.e., incorporating evidence from extinct and extant taxa), with emphasis on deliberate, incremental studies of nervous system form and function within and across vertebrata. Workshop participants were encouraged to reflect on those methods that are now standard in paleoneurology (e.g., the use of computed tomography (CT) to obtain digital endocasts and study endocranial morphology in extinct taxa), as well as to consider what methods and approaches might improve the scope of paleoneuro-

\section{KARGER}

() 2018 S. Karger AG, Basel

E-Mail karger@karger.com

www.karger.com/bbe logical studies (e.g., the application of cutting-edge comparative statistics, the use of novel structural and functional neuroimaging techniques on a more diverse array of extant taxa, and intentional coordination of paleontologists and neontologists in such efforts). The special issue of $B B E$ presented here offers papers authored by workshop presenters and their collaborators. In keeping with topics listed above, each paper considers endocast reconstruction and study, relevant application of modern comparative methods, and/or insights from novel imaging techniques.

The volume opens with studies that exemplify the application of digital technology in obtaining virtual endocasts, which permit inference and analysis of brain morphology for extinct taxa. Such papers demonstrate the high level of observable detail on endocasts from widely divergent vertebrate clades, including the enigmatic Oviraptorosauria (Late Cretaceous maniraptoran dinosaurs; authors: Amy M. Balanoff, Mark A. Norell, Aneila V.C. Hogan, and Gabriel S. Bever) to Hominini (Homo and earlier, close relatives; author: Emiliano Bruner). It is clear from these studies and others like them that endocasts offer a wealth of information about endocranial morphology and its evolution through deep time. However, what is also clear - especially to those who work di- 
rectly with endocasts - is that the ability to make biological inferences and track evolutionary trends using endocasts is hindered when: (a) the amount, complexity, and fluidity of newly available phylogenetic comparative methods (PCM) appears daunting, and (b) certain morphological, developmental, and/or functional aspects of relevant brain regions are poorly understood due to gaps in the neontological literature. Therefore, the deliberate formation of collaborations among paleoneurologists, computational PCM workers, and comparative neuroscientists would likely increase the scope and effectiveness of current endocast studies.

In the spirit of highlighting the potential usefulness of new methods and collaborations, the second half of this volume introduces some statistical and neuroimaging techniques that offer practical solutions for reducing the limitations on endocast studies. Haley D. O'Brien outlines and provides examples for how analyses of trait-dependent diversification may be augmented with fossil evidence, thereby allowing for more accurate and thorough identification of neuromorphological key innovations across the evolutionary history of a clade. Next, in their paper, David C. Van Essen, Chad J. Donahue, and Matthew F. Glasser review the use of connectomes to assess the organization, development, and evolution of cerebral and cerebellar cortices of the human brain. Then, James K. Rilling and Martijn P. van den Heuvel discuss in their paper how connectomes may be applied in a comparative framework to establish cortical homologies and novelties across and within modern primate species. Finally, Paul M. Gignac and Nathan J. Kley introduce a new application of diffusible iodine-based contrast-enhanced CT (diceCT) for visualizing, isolating, and analyzing brain structures (e.g., nuclei and white matter), and they demonstrate how diceCT can produce high-throughput datasets for broad neuroanatomical studies of extant vertebrates.
As workshop organizer and guest editor of this special issue, I hope that the topics covered here offer a wideangle view of the current standards and future directions for paleoneurological studies. I also hope that the discussions and papers related to the workshop will spark and foster new collaborative efforts among paleoneurologists, PCM workers, and neuroscientists. Such efforts may unlock the full potential of endocast studies and offer a more comprehensive understanding of the evolution of the vertebrate brain.

It has been a great pleasure to organize the 29th annual Karger Workshop and its subsequent special issue. Such endeavors would not have been possible without the excellent support, engagement, creativity, and productivity of all the presenters and authors who contributed to the workshop and this special $B B E$ issue, and I sincerely thank them for their efforts. I am also grateful to $S$. Karger AG and the J.B. Johnston Club for Evolutionary Neuroscience (JBJC) for supporting the workshop and providing authors with the opportunity to present, interact, and publish among such stimulating company. Thanks go also to Georg Striedter (BBE Editor) for offering guidance and advice throughout the development and execution of the workshop and special issue. Gratitude is extended to Elizabeth Catania (JBJC Principle Executive Officer) for advice and for facilitating and organizing many logistic details related to the workshop, and to the 2017 program committee members - Naoyuki Yamamoto, Kara Yopak, and Paul M. Forlano - for approving and supporting this workshop. I am grateful to both Amy L. Bauernfeind and Catherine M. Early for initial discussions of workshop theme development. Many thanks are also due to the expert colleagues who offered their time and insights while reviewing the special issue manuscripts. And, finally, all workshop attendees are gratefully appreciated for fruitful discussions. 